\title{
Extracorporeal membrane oxygenation for takotsubo cardiomyopathy that developed after mitral valve replacement
}

\author{
Jeong-Hyun Choi', In Duk Oh', Eunsil Shin', Sangho Lee', Jong-Mi Jeon', Hyung-Tak Kim², Hyo-Chul Youn ${ }^{3}$ \\ ${ }^{1}$ Department of Anesthesiology and Pain Medicine, Kyung Hee University College of Medicine, Seoul; ${ }^{2}$ Department of Anesthesiology and Pain Medicine, \\ Seoul Sungsim General Hospital, Seoul, ${ }^{3}$ Department of Thoracic and Cardiovascular Surgery, Kyung Hee University School of Medicine, Seoul, Korea
}

Takotsubo cardiomyopathy is a transient systolic and diastolic left ventricular dysfunction that presents several wall-motion abnormalities, while the coronary artery shows normal findings. Because patients with Takotsubo cardiomyopathy present with symptoms similar to acute coronary syndrome, the initial diagnosis and treatment are often difficult. The condition is often precipitated by acute emotional or physical stress and frequently occurs in postmenopausal women. Takotsubo cardiomyopathy may also occur in the perioperative period after cardiac and noncardiac surgery; surgery-associated Takotsubo cardiomyopathy reportedly accounts for 3\%-23\% of all cases. Of these perioperative cases, cardiothoracic surgery accounted for 16\%. However, few cases have been reported in patients undergoing cardiac surgery and managed with extracorporeal membrane oxygenation (ECMO). We report a case of Takotsubo cardiomyopathy managed with ECMO in a patient in the intensive care unit after mitral valve replacement.

Key Words: extracorporeal membrane oxygenation; intensive care units; Takotsubo cardiomyopathy

Takotsubo cardiomyopathy is a transient systolic and diastolic left ventricular dysfunction involving various wall-motion abnormalities, while the coronary artery shows normal findings [1]. The name was first described in the Japanese literature in 1990 and derived from the shape of a Japanese ceramic pot (called a takotsubo); it describes the unique pattern of the left ventricle, characterized by apical ballooning and a hyperkinetic base. It is often precipitated by acute emotional or physical stress and frequently occurs in women in the postmenopausal period. This disease is also known as stress-induced cardiomyopathy, Takotsubo syndrome, apical ballooning syndrome, or broken heart syndrome. It typically improves within 3 to 6 months. Although several hypotheses have been proposed for the etiology, catecholamineinduced microvascular dysfunction is now the most widely accepted cause [2].

Takotsubo cardiomyopathy may occur in the perioperative period after cardiac and noncardiac surgery. However, few cases have been reported in patients who have undergone cardiac surgery and were managed with extracorporeal membrane oxygenation (ECMO). We report a case of Takotsubo cardiomyopathy managed with ECMO that occurred in a patient in the intensive care unit (ICU) after mitral valve replacement.

\section{Case Report}

Received: September 4, 2018

Revised: October 17, 2018

Accepted: October 22, 2018

Corresponding author Jeong-Hyun Choi

Department of Anesthesiology and Pain Medicine, Kyung Hee University Hospital, 23 Kyungheedae-ro, Dongdaemun-gu, Seoul 02447, Korea

Tel: $+82-2-958-8589$

Fax: +82-2-958-8580

E-mail: choikhang@gmail.com

Copyright (๑) 2020 The Korean Society of Critical Care Medicine

This is an Open Access article distributed under the terms of Creative Attributions Non-Commercial License (https:// creativecommons.org/li-censes/by-nc/4.0/) which permits unrestricted noncommercial use, distribution, and reproduction in any medium, provided the original work is properly cited. 


\section{CASE REPORT}

A 52-year-old woman with rheumatic heart disease and atrial fibrillation was scheduled to undergo a mitral valve replacement and Maze operation for severe mitral stenosis. She had a history of decompressive craniectomy due to cerebral swelling and right middle cerebral artery infarction 1 year prior, and sequela included persistent weakness of her left side. Preoperative transthoracic echocardiography revealed normal left ventricular systolic function (ejection fraction, 63\%) and no regional wall motion abnormalities. Anesthesia induction and maintenance were performed as usual, and routine cardiac anesthesia monitoring was conducted. The mitral valve replacement was a 27-mm St. Jude Medical valve prosthesis (St. Jude Medical, Saint Paul, MN, USA), and the Maze operation proceeded well under cardiopulmonary bypass (CPB). Myocardial protection was performed via an infusion of cold cardioplegia via the aortic root cannula. Intraoperative transesophageal echocardiography showed no paravalvular leakage. Immediately after an aortic cross clamp, the cardiac activity was converted to normal sinus rhythm but returned to atrial fibrillation after a few minutes. Left ventricular systolic function remained normal. The patient was weaned from the CPB. After the operation, the patient was transferred to the ICU. The patient's vital signs were controlled through administration of dobutamine and norepinephrine, and tachycardia was controlled through amiodarone. The patient's blood pressure was maintained at 110/60 $\mathrm{mm} \mathrm{Hg}$, and her oxygen satu- ration was greater than $97 \%$. Extubation was performed the day after the operation. However, at 2 days postoperatively, the patient complained of dyspnea and chest discomfort, and electrocardiogram (ECG) revealed a premature ventricular complex. Transthoracic echocardiography revealed decreased left ventricular ejection fraction to $26 \%$ and akinesia of the middle and apical wall of the left ventricle (Figure 1). Takotsubo cardiomyopathy was suspected, but coronary artery disease could not be completely ruled out. On the 4 th postoperative day, the patient developed pulmonary edema and hypoxemia. The patient's systolic blood pressure was $80 \mathrm{~mm} \mathrm{Hg}$, and heart rate was 150 beats/min. The ECG showed atrial fibrillation with a rapid ventricular response. The doses of norepinephrine, dobutamine, and epinephrine were continuously increased. Transthoracic echocardiography showed a continuously worsening ejection fraction. The patient was intubated, and we decided to perform ECMO insertion because coronary angiography and management were not possible at the time due to the patient's condition. The results of laboratory tests were as follows: creatine kinase-MB, $1.5 \mu \mathrm{g} / \mathrm{L}$; troponin I, $1.73 \mathrm{ng} / \mathrm{ml}$; and brain natriuretic peptide (BNP), 4,073 pg/ml. The ECG showed atrial fibrillation with ST and T wave abnormality. The QT interval was $266 \mathrm{~ms}$. Vasopressor support with norepinephrine was continued. Coronary angiography was performed under ECMO, and the results were normal. Thus, Takotsubo cardiomyopathy was confirmed, and conservative treatment and supportive care were maintained. At 11 days postoperatively, the ECG showed QT prolongation (582 ms).
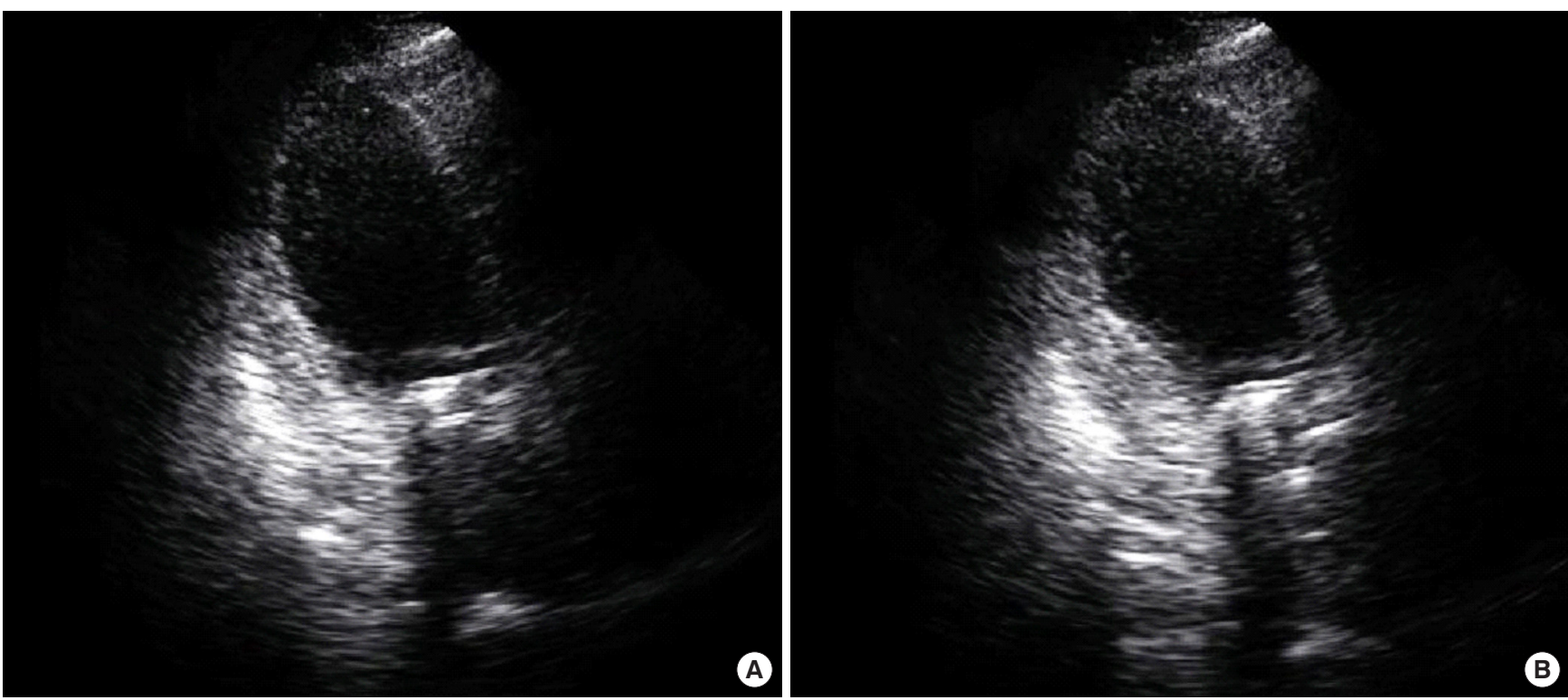

Figure 1. Apical view of the left ventricle with akinesia of the apical and mid segments on transthoracic echocardiography. (A) Diastole. (B) Systole. 

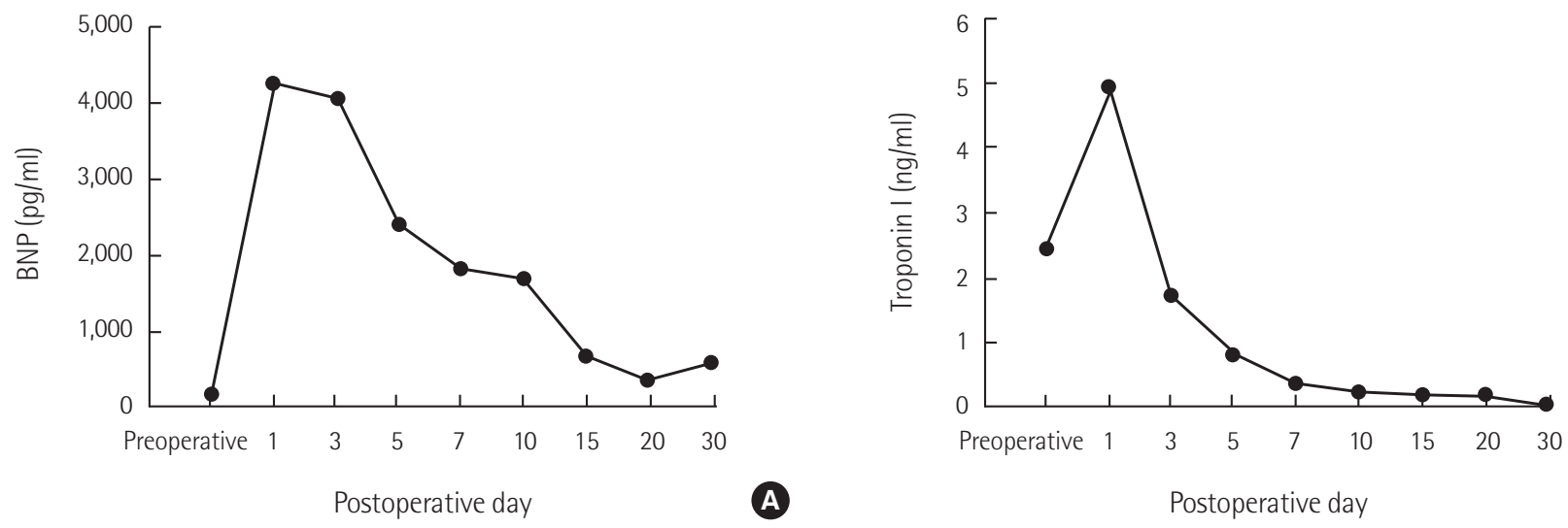

B

Figure 2. Changes in brain natriuretic peptide (BNP) (A) and troponin I (B) levels.

At 21 days postoperatively, transthoracic echocardiography revealed that the left ventricular ejection fraction had recovered to $38 \%$, and the ECMO was subsequently removed. Extubation was performed at 30 days postoperatively, and the patient was transferred to the general ward at 33 days postoperatively. At 35 days postoperatively, the patient's left ventricular ejection fraction had recovered to $58 \%$. The changes in $\mathrm{BNP}$ and serum troponin I levels are shown in Figure 2.

The patient was discharged on the 60th postoperative day after receiving rehabilitation for left-sided weakness. One year after the operation at a follow-up visit, the patient was without any complications and medicated with digitalis, diuretics, and warfarin.

\section{DISCUSSION}

Takotsubo cardiomyopathy is an acute and usually reversible heart failure syndrome. Because patients with Takotsubo cardiomyopathy present with symptoms similar to those seen in acute coronary syndrome, initial diagnosis and treatment of this condition are difficult. Careful examination is needed to confirm the diagnosis of Takotsubo cardiomyopathy; the condition has typical characteristics that need to be specifically checked. New diagnostic criteria for Takotsubo cardiomyopathy were created by the Heart Failure Association (HFA) of the European Society of Cardiology in 2015 [3]. The criteria are as follows: (1) transient regional wall-motion abnormalities, (2) wall-motion abnormalities typically extend beyond a single epicardial vascular distribution, (3) absence of atherosclerotic coronary artery disease, (4) new and reversible ECG abnormalities, (5) significantly elevated serum BNP or natriuretic peptide-proBNP during the acute phase, (6) positive but relatively small elevation in cardiac troponin, and (7) recovery of ventricular systolic function (3-6 months). Our patient had transient akinesia of the mid and apical left ventricle, normal coronary angiography, QT prolongation, elevated serum BNP and troponin levels, and recovery of left ventricular systolic function within 1 month. Therefore, the patient's clinical data adhere to the diagnostic criteria mentioned above. However, ECG changes and BNP level may take 6-12 months to recover and may persist abnormally in some cases [1].

If a patient has at least one major risk factor or two or more minor risk factors among the risk factors suggested by the HFA, the patient is classified as a high-risk patient [3]. Our patient was classified as a high-risk patient because she had two major risk factors (pulmonary edema and left ventricular ejection fraction $<35 \%$ ) as well as two minor risk factors (QT prolongation $\geq 500 \mathrm{~ms}$ and $\mathrm{BNP} \geq 600 \mathrm{pg} / \mathrm{ml}$ ). Management by a cardiologist and ICU care are essential for treating these patients. Serious complications of Takotsubo cardiomyopathy include congestive heart failure, pulmonary edema, and cardiogenic shock. It is reported that patients experiencing cardiogenic shock had a much higher mortality [4]. These high risk patients often require ventilatory support, advanced cardiovascular support, and even cardiopulmonary resuscitation. To the best of our knowledge, there has been no randomized trial to define the optimal management of patients diagnosed with Takotsubo syndrome. Although a number of authors favor the use of an intra-aortic balloon pump to minimize or avoid inotropic therapy, the expert consensus suggests that the use of ECMO or left ventricular assist devices for patients with cardiogenic shock associated with Takotsubo cardiomyopathy yields better outcomes [5]. In our patient, we were able to diagnose Takotsubo cardiomyopathy early using transthoracic echocardiography and laboratory tests. Moreover, early application of ECMO enabled rapid recovery of cardiac function. 
Takotsubo cardiomyopathy is triggered by emotional or physical stress. Takotsubo cardiomyopathy associated with surgery is reported to account for $3 \%-23 \%$ of all cases, and cardiomyopathy developing after cardiothoracic surgery accounted for $16 \%$ of these perioperative cases [6-8]. Patients suffer emotional stress at the prospect of undergoing a major surgery, and they also experience increased catecholamines by direct stimulation of pain. Patients undergoing cardiac surgery are particularly vulnerable to cardiac toxicity due to catecholamines since the heart is directly stimulated during surgery. Plasma catecholamine concentration is significantly elevated in patients with Takotsubo cardiomyopathy compared to levels in patients with other cardiac conditions, such as acute myocardial infarction. In our case, the psychological stress due to ICU admission and the maintenance of supportive catecholamines after surgery could be the causes of the disease. In addition, there is a report that a cerebrovascular event is associated with Takotsubo cardiomyopathy [9]. Therefore, this patient may have had an increased risk of developing Takotsubo cardiomyopathy because she had a history of a cerebrovascular event. To prevent development of Takotsubo cardiomyopathy after surgery, it is important to reduce the psychological and physical stress on the patient through aggressive pain control and adequate sedation. An agent such as dexmedetomidine, which controls pain and blunts the sympathetic nervous system, may be an alternative [10].

A large retrospective study of 26 centers in the United States and Europe found that systolic dysfunction is classified into four types: apical (81.7\%), midventricular (14.6\%), basal (2.2\%), and focal (1.5\%) [1]. Our patient was found to have the typical apical type. Although the dysfunction is named Takotsubo due to its distinctive shape, other types of systolic dysfunctions, in addition to the typical type, should be kept in mind.

In conclusion, early diagnosis of postsurgical Takotsubo cardiomyopathy with echocardiography and laboratory testing is important for successful patient management. If cardiac function has severely deteriorated, the application of ECMO can enable patient recovery.

\section{CONFLICT OF INTEREST}

No potential conflict of interest relevant to this article was reported.

\section{ORCID}

Jeong-Hyun Choi https://orcid.org/0000-0003-1995-1220 $\begin{array}{ll}\text { In Duk Oh } & \text { https://orcid.org/0000-0001-5372-0843 } \\ \text { Eunsil Shin } & \text { https://orcid.org/0000-0003-0939-3704 } \\ \text { Sangho Lee } & \text { https://orcid.org/0000-0001-5943-7715 } \\ \text { Jong-Mi Jeon } & \text { https://orcid.org/0000-0002-5169-8563 } \\ \text { Hyung-Tak Kim } & \text { https://orcid.org/0000-0003-4509-0751 } \\ \text { Hyo-Chul Youn } & \text { https://orcid.org/0000-0002-6645-1643 }\end{array}$

\section{AUTHOR CONTRIBUTIONS}

Conceptualization: JHC. Data curation: IDO, ES, HTK. Formal analysis: IDO, JHC. Methodology: JHC. Project administration: JHC. Visualization: SL, JMJ. Writing - original draft: IDO, ES, HTK. Writing - review \& editing: JHC, HCY.

\section{REFERENCES}

1. Templin C, Ghadri JR, Diekmann J, Napp LC, Bataiosu DR, Jaguszewski M, et al. Clinical features and outcomes of Takotsubo (stress) cardiomyopathy. N Engl J Med 2015;373:929-38.

2. Wittstein IS, Thiemann DR, Lima JA, Baughman KL, Schulman SP, Gerstenblith G, et al. Neurohumoral features of myocardial stunning due to sudden emotional stress. N Engl J Med 2005;352:539-48

3. Lyon AR, Bossone E, Schneider B, Sechtem U, Citro R, Underwood SR, et al. Current state of knowledge on Takotsubo syndrome: a Position Statement from the taskforce on Takotsubo Syndrome of the Heart Failure Association of the European Society of Cardiology. Eur J Heart Fail 2016;18:8-27.

4. Stiermaier T, Eitel C, Desch S, Fuernau G, Schuler G, Thiele H, et al. Incidence, determinants and prognostic relevance of cardiogenic shock in patients with Takotsubo cardiomyopathy. Eur Heart J Acute Cardiovasc Care 2016;5:489-96.

5. Bonacchi M, Maiani M, Harmelin G, Sani G. Intractable cardiogenic shock in stress cardiomyopathy with left ventricular outflow tract obstruction: is extra-corporeal life support the best treatment? Eur J Heart Fail 2009;11:721-7.

6. Hessel EA 2nd. Takotsubo cardiomyopathy and its relevance to anesthesiology: a narrative review. Can J Anaesth 2016;63: 1059-74.

7. Pergolini A, Zampi G, Casali G, Madeo A, Visconti CL, Cipullo PL, et al. Takotsubo syndrome after mitral valve replacement: case report and brief review of the literature. J Cardiothorac Vasc Anesth 2015;29:431-5.

8. Chiariello GA, Bruno P, Colizzi C, Crea F, Massetti M. Takotsubo cardiomyopathy following cardiac surgery. J Card Surg 2016;31:89-95.

9. Morris NA, Chatterjee A, Adejumo OL, Chen M, Merkler AE, 
Murthy SB, et al. The risk of Takotsubo cardiomyopathy in acute neurological disease. Neurocrit Care 2019;30:171-6.

10. Mukhtar A, Moharam H, Sarhan K, Hosni H, Salah M. Liver transplantation using dexmedetomidine in a patient with a history of Takotsubo cardiomyopathy. A A Case Rep 2016;6: 14-6. 